\title{
Theoretical coupling longitudinal-transverse model and experimental verification of transverse vibration of rope for multi-rope friction hoisting system
}

\author{
Juan Wu ${ }^{1,2} \cdot$ Ziming Kou ${ }^{1,2}$
}

Received: 10 April 2015/Revised: 16 October 2015/ Accepted: 26 October 2015/Published online: 24 February 2016

(C) The Author(s) 2016. This article is published with open access at Springerlink.com

\begin{abstract}
Using the mass of time-varying length balance rope focused on the hoisting conveyance, the coupling longitudinal-transverse model of mine friction hoist was established by using of the Hamilton's principle. The modified Galerkin's method was used to discretize partial differential Eqs. The mine hoisting system was used to the example to analysis the relation between the load, velocity and transverse vibration of rope. The in situ tests were illustrated to evaluate the proposed mathematical model. The results showed that the modeling method can well represent the transverse vibration of rope.
\end{abstract}

Keywords Hoisting system $\cdot$ Vibration $\cdot$ Rope $\cdot$ Longitudinal-transverse $\cdot$ Coupling

\section{Introduction}

Due to their ability to resist relatively large axial loads, ropes have been widely used in many different applications to support structures, conduct signals, and carry payloads. In this latter application, cables have played a vital role in the vertical transport systems such as elevators (Zhu and $\mathrm{Ni}$ 2000; Zhu et al. 2001; Zhu and Chen 2005; Zhang 2007), cranes and particularly mine friction hoists (Kaczmarczyk and Ostachowicz 2003a; Sandilo and van Horssen 2014).

A common arrangement of mine friction hoist system is shown in Fig. 1. It comprises a driving friction wheel mounted in headgear, four or six hoist ropes, three balance ropes and two conveyances. In this system, the hoist ropes form vertical ropes connecting the friction wheel and conveyances. This configuration exhibits variable-length and transport speed during operation. It is classified in the

Juan Wu

wujuanz@163.com

1 Mechanical Engineering College, Taiyuan University of Technology, Taiyuan 030024, China

2 Shanxi Provincial Engineering Laboratory for Mine Fluid Control, Taiyuan 030024, China category of one-dimensional continuous systems and consequently the displacement in situ depends on the time and a single spatial coordinate.

Elevator cables and friction hoist ropes are classified in the category of one-dimensional continuous systems. The vibrations of elevator ropes had been widely studied (Zhu and Ni 2000; Zhu et al. 2001; Zhu and Chen 2005; Zhang 2007; Sandilo and van Horssen 2014). Zhu and Ni (2000) generally investigated the stability characteristics of horizontally and vertically translating strings and beams with arbitrarily varying-length and with various boundary conditions. The forced response of translating media with variable length and tension was analyzed by Zhu and Chen (2005). An active control methodology using a point-wise control force and/or moment was developed by Zhu et al. to dissipate the vibratory energy of a translating medium with arbitrarily varying length. Zhang (2007) established the vibration governing Eq.s and energy Eq.s of coupled transverse-longitudinal vibration of elevator cables. Sandilo and van Horssen (2014) used a multiple-timescale perturbation method to construct formal asymptotic approximations of the solutions to show the complicated dynamical behavior of the string.

The longitudinal vibrations of ropes in mine hoist have been widely studied by many researchers. The single-rope 


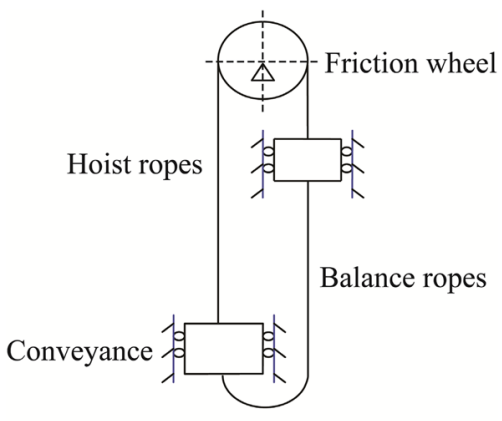

Fig. 1 A friction hoisting installation

mine drum is the catenary-vertical rope system and the coupled lateral-longitudinal dynamic response of the cables was studied by Kaczmarczyk and Ostachowicz (2003a, 2003b). Qin (2007) analyzed the longitudinal vibration of hoisting ropes by using the Lagrange principle. Li (2010) established Horizontal and vertical vibration models of the hoisting container under the steel guide and the vibration characteristic of hoisting container under different faults of steel guide was studied. To master the impact dynamical behaviors of the hoisting rope during the skip loading, the mechanical model of the coupled longitudinal-torsional vibration for hoisting rope were built by Cao (2010).

The importance of vibrations for ropes cannot be overestimated, especially in the context of the mining industry, where the safety of personnel and profitability rely on stable designs (Kaczmarczyk and Ostachowicz 2003). However; few transverse vibrations of ropes on coupling longitudinal-transverse model for mine hoist systems have been reported.

In this paper, the coupling longitudinal-transverse governing Eqs. of motion were established. The transverse dynamic responses of conveyance and ropes during descending cycle and ascending cycle were especially investigated. The in situ tests were performed to evaluate the proposed mathematical model.

\section{The governing equations of motion}

The model was established on the following assumptions: (1) Young's modulus $E$, cross section area $A$, and mass per unit length were always constants; (2) The bending stiffness of rope and influence of air current were ignored; (3) The hoist ropes were simplified as single rope with the equivalent cross-section area. The rope was modeled as a taut string; (4) The mass of time-varying balance ropes was focused on conveyance.

A model of the mine hoist was shown in Fig. 2. The hoisting container was modeled as a rigid body of mass $m$

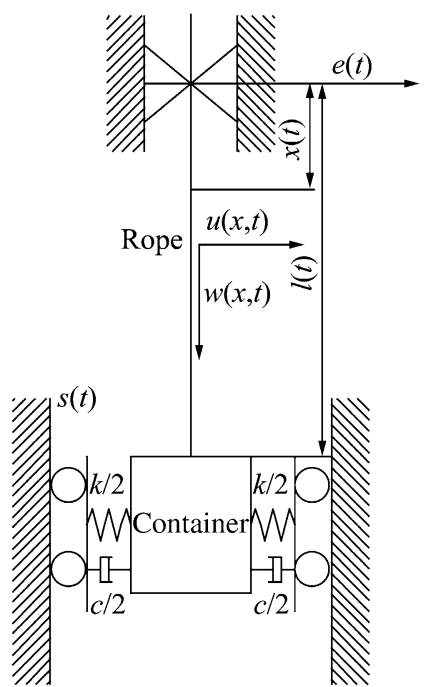

Fig. 2 A model of mine hoists system

attached at the lower end $x=l(t)$. The hoisting container was supported by a guide of stiffness $k$ and damping coefficient $c$. The suspension of the container against the guide rails was assumed to be rigid. During its motion, the hoisting rope of mass per unit length $\rho$ had a variable length $l(t)$ and an axial velocity $V=\dot{l}(t)$ where the over dot denoted time differentiation. The balance rope of mass per unit length $\rho_{2}$ had a variable length $H-l(t)$, where $H$ was the hoisting height.

The transverse displacement of the rope particle instantaneously located at spatial position $x$ at time $t$ was described by $u(x, t)$ and longitudinal displacement was described by $w(x, t)$. There was a boundary excitation $e(t)$ in the upper end and the rigid roughness of the guide' surface was denoted by $s(t)$.

\subsection{Free motion with damping}

The Hamilton principle applied to the mine hoists yielded

$\int_{t_{1}}^{t_{2}}\left(\delta T-\delta E_{e}-\delta E_{g}+\delta W\right) \mathrm{d} t=0$,

where $T, E_{e}, E_{g}$ and $W$ denoted the system kinetic energy, the rope elastic strain energy, the system gravitational potential energy and the damping force energy, respectively. The kinetic energy of the system was expressed as

$T=\frac{1}{2} m_{e} V_{c}^{2}+\frac{1}{2} \rho \int_{0}^{l} V^{2} \mathrm{~d} x$,

where $m_{e}=m+(H-l(t)) \rho_{2}, V_{c}$ and $V$ represented the total velocity at the conveyance and $x$ on rope, respectively. 
The elastic strain energy of the rope was

$E_{e}=\frac{1}{2} k[u(l(t), t)]^{2}+\int_{0}^{l}\left(P \varepsilon+\frac{1}{2} E A \varepsilon^{2}\right) \mathrm{d} x$,

where $E A$ represented the tensile stiffness of the rope, $\varepsilon$ and $P$ represented the strain measure in $x$ on rope and the quasistatic tension of rope.

$P=\left[m_{e}+\rho(l(t)-x)\right](g-a)$,

$\varepsilon=w_{x}+\frac{1}{2} w_{x}^{2}+\frac{1}{2} u_{x}^{2}$

where the subscript ()$_{x}$ denoted the partial differentiation with respect to $x, a$ and $g$ represented the acceleration and gravitational acceleration

The gravitational potential energy of the cable was given by

$E_{g}=E_{g s}-\int_{0}^{l} \rho g w(x, t) \mathrm{d} x-m_{e} g w_{c}(l, t)$,

where $E_{g s}$ was the gravitational potential energy in the undeformed reference configuration.

The damping force energy was

$W=\left(-c \frac{D y(l(t), t)}{D t}+k s+c \dot{s}\right) u(l(t), t)$.

And the boundary conditions were

$$
\begin{aligned}
& w(0, t)=0 \quad \delta w(0, t)=\delta w\left(x, t_{1}\right)=\delta w\left(x, t_{2}\right)=0 \\
& u(0, t)=0
\end{aligned}
$$

The governing Eqs. for the system were yielded

$$
\begin{aligned}
& \rho\left(w_{t t}+2 v w_{x t}+\dot{v} w_{x}+v^{2} w_{x x}+\dot{v}\right)-P_{x}\left(1+w_{x}\right) \\
& \quad-P w_{x x}-\rho g-E A\left(w_{x x}+3 w_{x} w_{x x}+\frac{3}{2} w_{x}^{2} w_{x x}\right. \\
& \left.\quad+u_{x} u_{x x}+w_{x} u_{x} u_{x x}+\frac{1}{2} u_{x}^{2} w_{x x}\right)=0, \\
& \rho\left(u_{t t}+2 v u_{x t}+\dot{v} u_{x}+v^{2} u_{x x}\right)-P_{x} u_{x}-P u_{x x} \\
& -E A\left(w_{x} u_{x x}+u_{x} w_{x x}+\frac{1}{2} w_{x}^{2} u_{x x}+w_{x} u_{x} w_{x x}+\frac{3}{2} u_{x}^{2} w_{x x}\right)=0 .
\end{aligned}
$$

The boundary conditions at $x=l(t)$ were

$$
\begin{aligned}
& m_{e}\left(w_{t t}+2 v w_{x t}+\dot{v}+\dot{v} w_{x}+v^{2} w_{x x}\right) \\
& \quad+(P+E A \varepsilon)\left(1+w_{x}\right)-m g=0 \\
& m_{e}\left(u_{t t}+2 v u_{x t}+\dot{v} u_{x}+v^{2} u_{x x}\right)+(P+E A \varepsilon) u_{x} \\
& \quad+c\left(u_{t}+v u_{x}\right)+k u-k s-c \dot{s}=0
\end{aligned}
$$

In this non-linear system, Eq. (9a) described the longitudinal dynamics of rope and Eq. (9b) represented the transverse dynamics of rope.

\subsection{Boundary excitation}

The cable cross-over motion at the friction wheel resulted in a boundary excitation. The boundary condition was formulated as

$u(0, t)=e(t)$

This boundary was inhomogeneous boundary conditions. The function $u(x, t)$ with inhomogeneous boundary conditions was translated to the function $y(x, t)$ with homogeneous boundary condition,

$u(x, t)=y(x, t)+h(x, t)$,

where $h(x, t)=a_{0}(t)+a_{1}(t) \frac{x-x_{0}}{x_{1}-x_{0}}$.

Thus, when the excitation terms were taken into consideration, the response of the system was governed by

$$
\begin{gathered}
\rho\left(w_{t t}+2 v w_{x t}+\dot{v} w_{x}+v^{2} w_{x x}+\dot{v}\right)-P_{x}\left(1+w_{x}\right)-P w_{x x} \\
-E A\left(w_{x x}+3 w_{x} w_{x x}+\frac{3}{2} w_{x}^{2} w_{x x}+\left(y_{x}+h_{x}\right)\left(y_{x x}+h_{x x}\right)\right. \\
\left.+w_{x}\left(y_{x}+h_{x}\right)\left(y_{x x}+h_{x x}\right)+\frac{1}{2}\left(y_{x}+h_{x}\right)^{2} w_{x x}\right)-\rho g=0,
\end{gathered}
$$

$$
\begin{gathered}
\rho\left(y_{t t}+2 v y_{x t}+\dot{v} y_{x}+v^{2} y_{x x}\right)-P_{x} y_{x}-P y_{x x} \\
-E A\left(w_{x} y_{x x}+y_{x} w_{x x}+\frac{1}{2} w_{x}^{2} y_{x x}+w_{x} y_{x} w_{x x}\right. \\
\left.+\frac{3}{2}\left(y_{x}+h_{x}\right)^{2}\left(y_{x x}+h_{x x}\right)\right)=f(x, t) .
\end{gathered}
$$

where $f(x, t)=P_{x} h_{x}+P h_{x x}+E A\left(w_{x} h_{x x}+h_{x} w_{x x}+\frac{1}{2} w_{x}^{2} h_{x x}+\right.$ $\left.w_{x} h_{x} w_{x x}\right)-\rho\left(h_{t t}+a h_{x}+2 v h_{x t}+v^{2} h_{x x}\right)$.

The boundary conditions at $x=l(t)$ were the same with Eqs. (9a) and (9b).

\section{Numerical solutions}

\subsection{Spatial discretization}

The modified Galerkin's method was employed to discretize the governing partial differential equations. The instantaneous orthonormal eigenfunction with time-varying length $l(t)$ were used as the trial functions. They can be expressed as $\varphi_{j}(x, t)=\frac{1}{\sqrt{l(t)}} \varphi_{j}(\xi)(j=1,2,3 \ldots)$, where $\xi=\frac{x}{l(t)}, \varphi_{j}(\xi)=\sqrt{2} \sin \left(\frac{2 j-1}{2} \pi \xi\right)$.

The solutions of Eq. (13) were assumed in the form

$$
\begin{aligned}
& w(x, t)=\sum_{j=1}^{n} p_{j}(t) \varphi_{j}(x, t)=\frac{1}{\sqrt{l(t)}} \sum_{j=1}^{n} p_{j}(t) \varphi_{j}(\xi), \\
& y(x, t)=\sum_{j=1}^{n} q_{j}(t) \varphi_{j}(x, t)=\frac{1}{\sqrt{l(t)}} \sum_{j=1}^{n} q_{j}(t) \varphi_{j}(\xi) .
\end{aligned}
$$



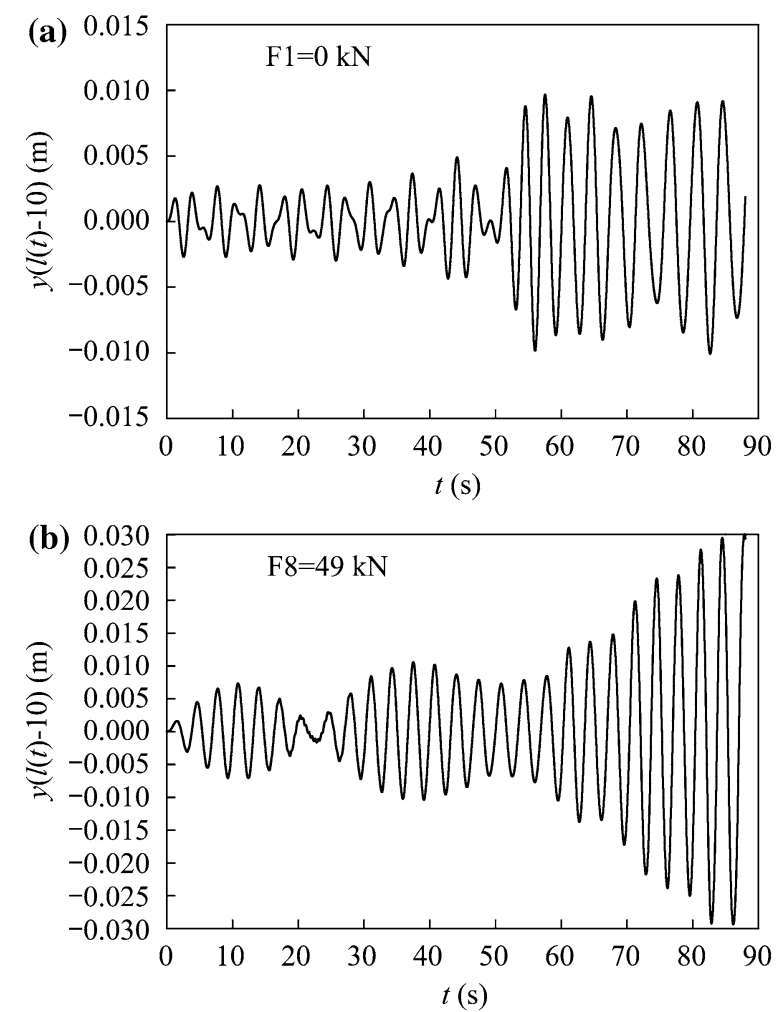

Fig. 3 The transverse response time histories of rope at $6 \mathrm{~m} / \mathrm{s}$ in descending cycle

where $p_{j}(t)$ and $q_{j}(t)$ were the generalized coordinates, and $n$ was the number of included modes. Substituting Eq. (14) into the governing Eq. (13) and boundary conditions of Eq. (9), multiplying the governing equation by $\frac{1}{\sqrt{l(t)}} \varphi_{j}(\xi)$, integrating it from $x=0$ to $l(t)$, yielded the discretized equations of motion.

$$
\begin{aligned}
& {\left[\begin{array}{cc}
M_{1} & 0 \\
0 & M_{2}
\end{array}\right]\left[\begin{array}{l}
P^{\prime \prime} \\
Q^{\prime \prime}
\end{array}\right]+\left[\begin{array}{cc}
C_{1} & 0 \\
0 & C_{2}
\end{array}\right]\left[\begin{array}{l}
P^{\prime} \\
Q^{\prime}
\end{array}\right]+\left[\begin{array}{cc}
K_{1} & 0 \\
0 & K_{2}
\end{array}\right]\left[\begin{array}{l}
P \\
Q
\end{array}\right]} \\
& =\left[\begin{array}{l}
C P_{1} \\
C P_{2}
\end{array}\right]+\left[\begin{array}{l}
F_{1} \\
F_{2}
\end{array}\right]
\end{aligned}
$$

where $P=\left[p_{1}, p_{2}, \ldots, p_{n}\right]^{T}$ and $Q=\left[q_{1}, q_{2}, \ldots, q_{n}\right]^{T}$ were the vectors of generalized coordinates, $M, C, K, C P$ and $F$ were symmetric mass, damping, stiffness matrices, coupling and generalized force matrices. Entries of these matrices were given in Appendix 1.

\subsection{Example and discussions}

The example of mine hoisting system was illustrated to evaluate the proposed mathematical model. The maximum
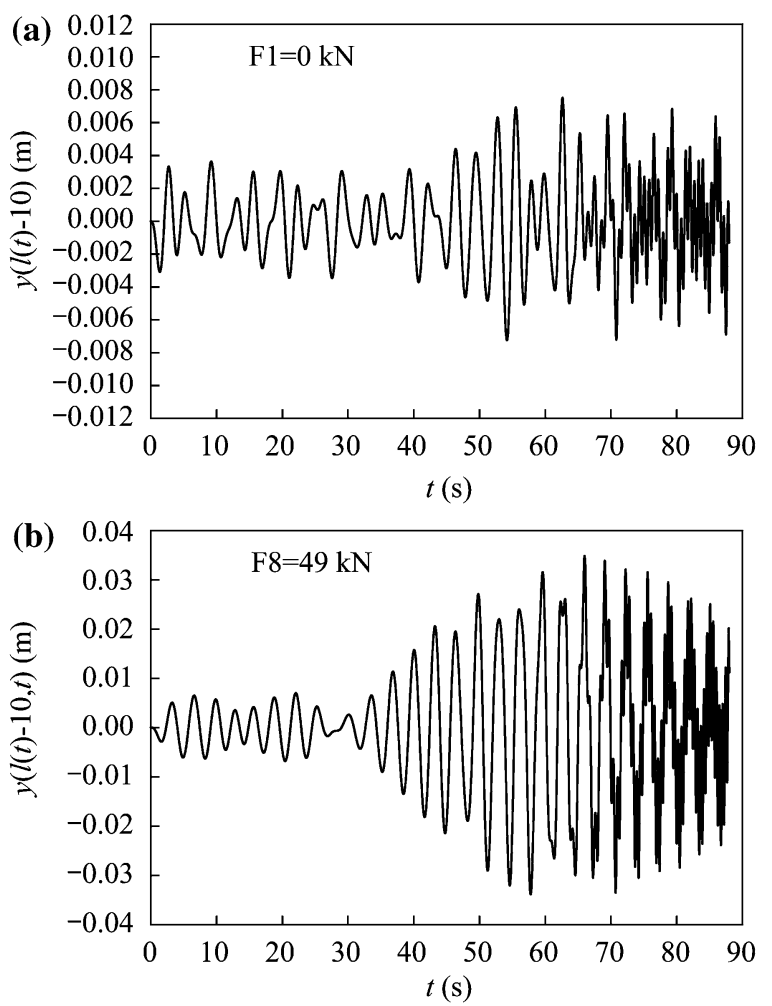

Fig. 4 The transverse response time histories of rope at $6 \mathrm{~m} / \mathrm{s}$ in ascending cycle

hoisting height and acceleration were $263 \mathrm{~m}$ and $0.6 \mathrm{~m} / \mathrm{s}^{2}$. The mass of container was $46000 \mathrm{~kg}$. There were six hoisting ropes and mass per unit length was $8.52 \mathrm{~kg} / \mathrm{m}$. And the mass per unit length of three balance ropes was $16.8 \mathrm{~kg} / \mathrm{m}$. The normal load range was $0,7,14,21,28,35$, 42 and $49 \mathrm{kN}$ and the speed was 6,7 and $8 \mathrm{~m} / \mathrm{s}$.

The prescribed displacement, velocity, acceleration curves were utilized as the input of Eq. (15) to obtain the longitudinal and transverse vibration response of rope and conveyance. The transverse vibration responses of rope were specifically analyzed in this paper. The boundary excitation $e(t)$ was represented by

$e(t)=0.001 \times \sin (1.45 \pi t)$

The $s(t)$ was given by

$s(t)=0.001 \times \sin (0.6 \pi t)$.

The transverse dynamic responses of rope at (F1) and (F8) were shown in Figs. 3 and 4. The vibration of rope in descending cycle was different from that in ascending cycle. In descending cycle, the amplitude of transverse dynamic responses was gradually increased with increasing the length of rope. In ascending cycle, the amplitude of 

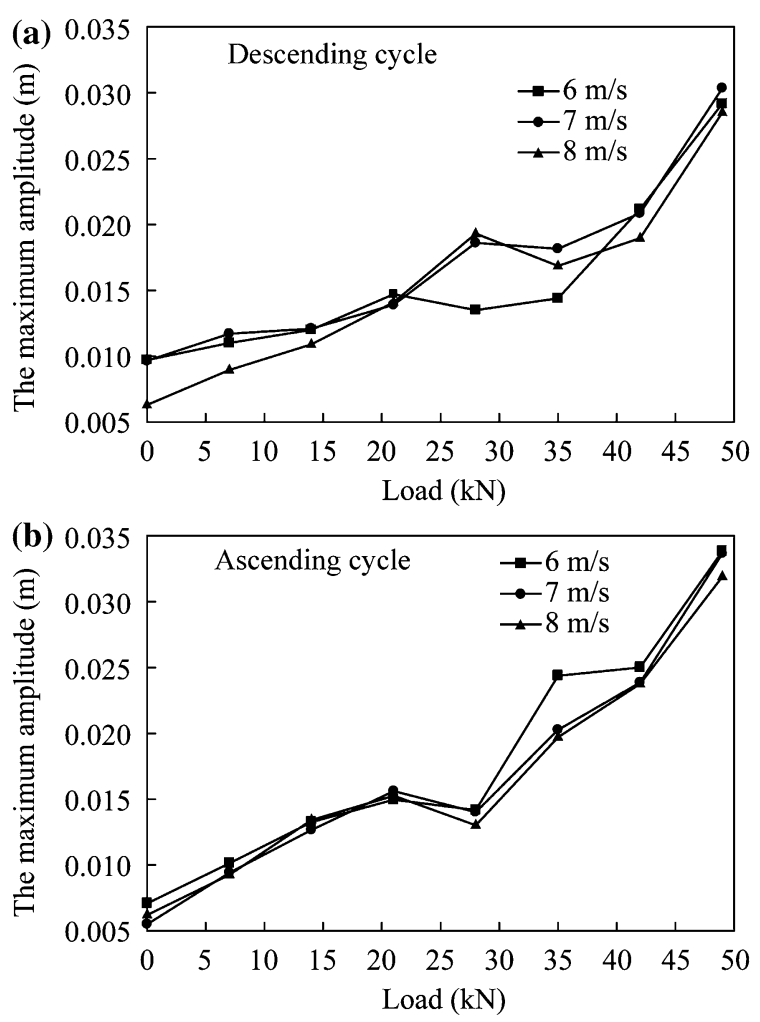

Fig. 5 Variations of maximum amplitudes of rope at different loads and velocities: $\mathbf{a}$ in descending cycle and $\mathbf{b}$ in ascending cycle

transverse dynamic responses was gradually increased with decreasing the length of rope and the frequency of transverse dynamic responses was higher near to the pithead.

Figure 5 showed the variations of maximum amplitude at different loads and velocities. Generally speaking, the maximum amplitudes in ascending and descending cycle showed the similar trend and increase with the increase of load. The maximum amplitudes in ascending cycle were the higher and those in descending cycle were lower. The maximum amplitudes showed a fluctuation with the increase of speed and the maximum amplitudes at $8 \mathrm{~m} / \mathrm{s}$ were lower than those at 6 and $7 \mathrm{~m} / \mathrm{s}$.

\section{In-situ tests}

Four INV9822 acceleration sensors were installed on the four ropes respectively with a distance of $10 \mathrm{~m}$ from the conveyance, shown in Fig. 6. The data acquisition analyzer

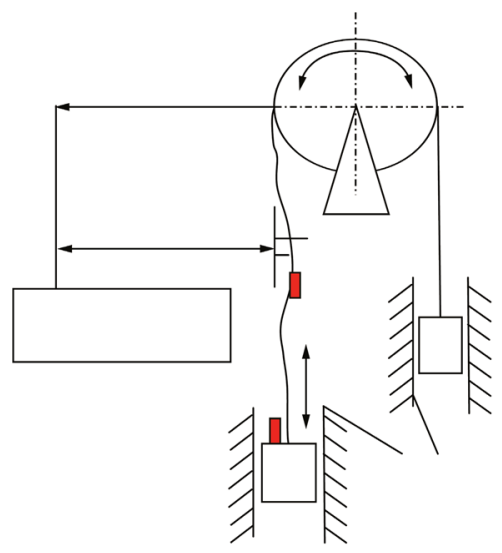

(a) Overall test set-up

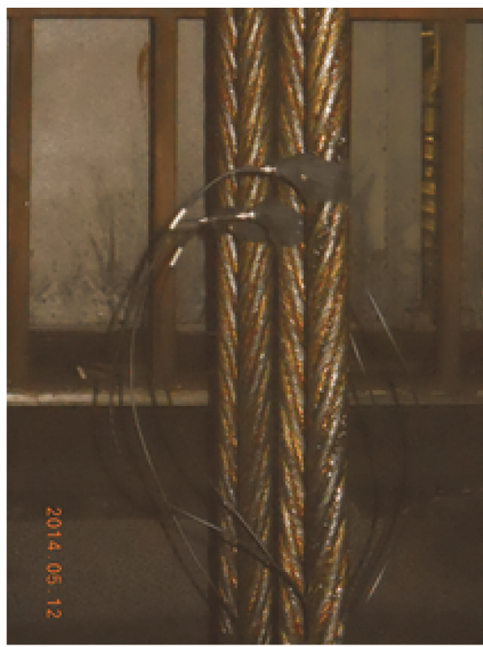

(b) Sensors on ropes

Fig. 6 Test setup

was analyzer INV3060S and the data acquisition and signal analysis platform was DASP-V10, which were made by the Dongfang Vibration and Noise Technology Institute, Beijing, China.

The history of response time of the rope for in situ tests was shown in Figs. 7, 8 and 9 was the amplitude-frequency characteristics of transverse vibration. Though the influence of complicated conditions usually occurred in in situ tests, boundary excitations in the upper end and the rigid roughness of the guide' surface would be the major factors. Compared the response time histories of rope and amplitude-frequency characteristic obtained by the present analysis with those obtained experimentally, the mathematical model and numerical solution were in an acceptable level of accuracy as 

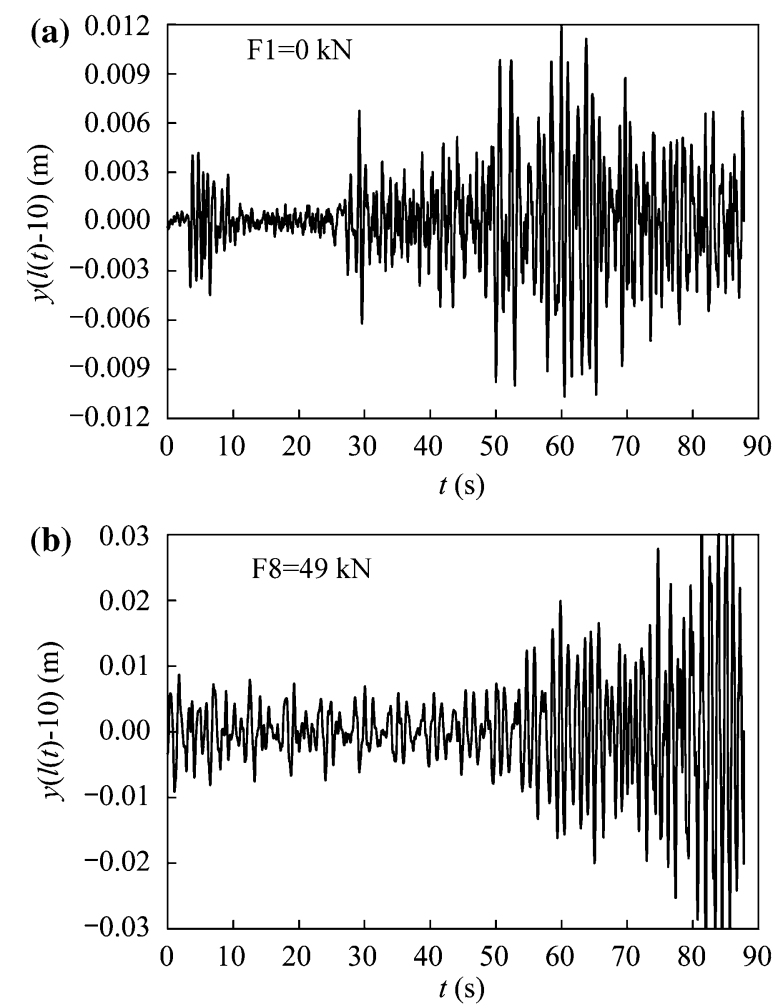

Fig. 7 The transverse response time histories of rope in descending cycle

compared with experimental data. It suggests that the models were validated.

\section{Conclusions}

(1) The Hamilton's principle was employed to study the coupling longitudinal-transverse governing equations of motion for the rope in mine hoisting system. The partial differential equations of motion of this system have been derived.

(2) The transverse vibration of rope had been theoretically studied. The transverse vibration of rope in ascending cycle was stronger than that in descending cycle. The maximum amplitudes in ascending and descending cycle showed the similar trend and increase with the increase of load. The maximum amplitudes at $8 \mathrm{~m} / \mathrm{s}$ were the lowest.

(3) The result obtained by the mathematical model was compared with result obtained experimentally. The present model was proved to be accurate sufficiently by compared with test results.

(4) The theoretical formulation and numerical solution scheme provided an efficient method for the analysis of the transverse vibration of the rope for mine hoist system.
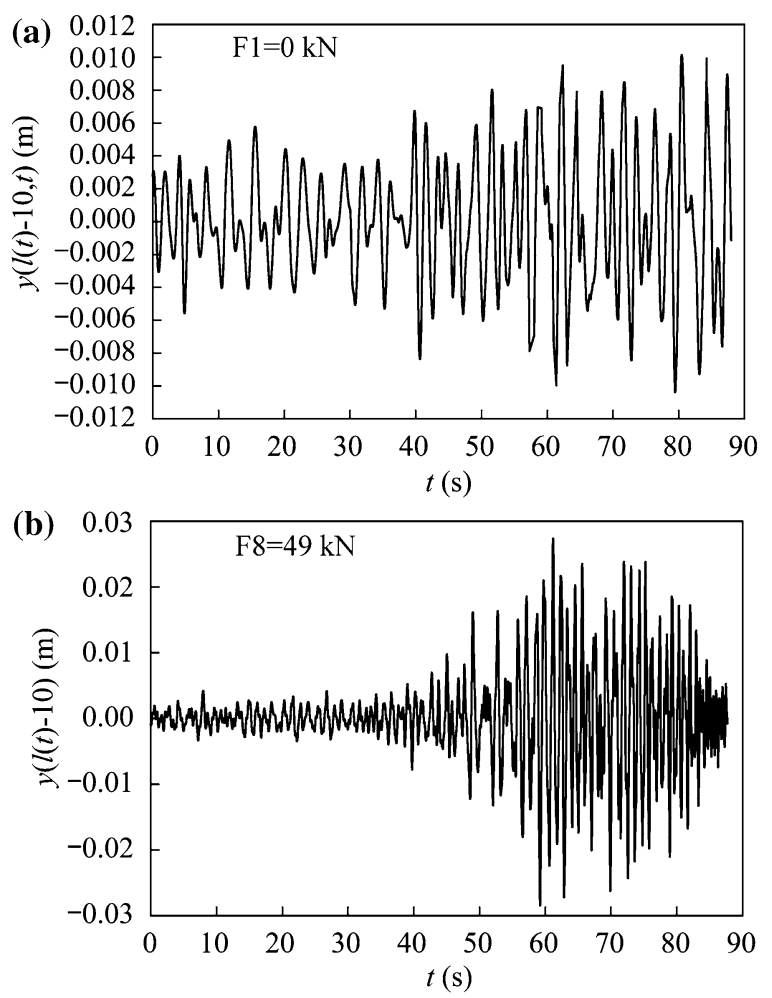

Fig. 8 The transverse response time histories of rope in ascending cycle
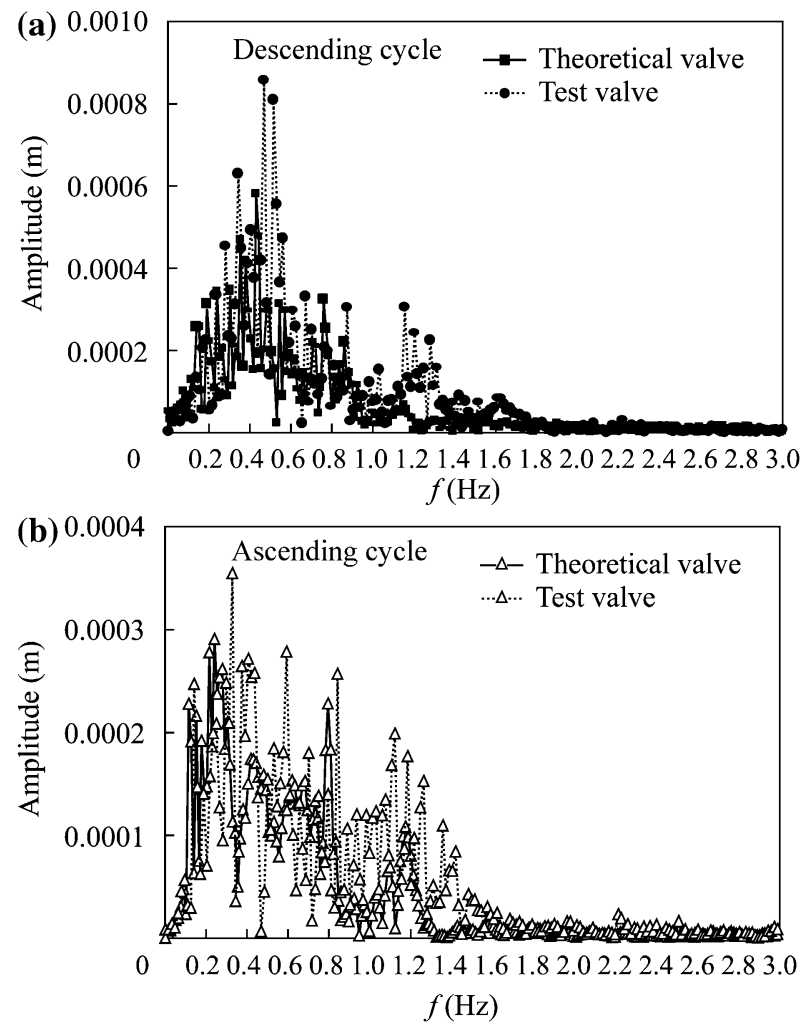

Fig. 9 The amplitude-frequency characteristic of transverse vibration 
Acknowledgments The authors are grateful to the International S \& T Cooperation Program of China (2011DFA72120) and NSFC (51205272) for financial support.

Open Access This article is distributed under the terms of the Creative Commons Attribution 4.0 International License (http://crea tivecommons.org/licenses/by/4.0/), which permits unrestricted use, distribution, and reproduction in any medium, provided you give appropriate credit to the original author(s) and the source, provide a link to the Creative Commons license, and indicate if changes were made.

\section{Appendix 1: Entries of matrices in Eq. (15)}

$$
\begin{aligned}
& m_{1 i j}=m_{2 i j}=\rho \delta_{i j}+m l^{-1} \varphi_{i}(1) \varphi_{j}(1) \\
& c_{1 i j}=-\rho v l^{-1} \delta_{i j} \\
& +\int_{0}^{1} 2 \rho v l^{-1}(1-\xi) \varphi_{i}(\xi) \varphi_{j}^{\prime}(\xi) \mathrm{d} \xi-m v l^{-2} \varphi_{i}(1) \varphi_{j}(1) \\
& c_{2 i j}=-\rho v l^{-1} \delta_{i j}+\int_{0}^{1} 2 \rho v l^{-1}(1-\xi) \varphi_{i}(\xi) \varphi_{j}^{\prime}(\xi) \mathrm{d} \xi \\
& -m v l^{-2} \varphi_{i}(1) \varphi_{j}(1)+c l^{-1} \varphi_{i}(1) \varphi_{j}(1) \\
& k_{1 i j}=\left(\frac{3 \rho}{4} v^{2} l^{-2}-\frac{\rho a}{2} l^{-1}\right) \delta_{i j} \\
& -3 \int_{0}^{1} \rho v^{2} l^{-2}(1-\xi) \varphi_{i}(\xi) \varphi_{j}^{\prime}(\xi) \mathrm{d} \xi \\
& +\int_{0}^{1} \rho a l^{-1}(1-\xi) \varphi_{i}(\xi) \varphi_{j}^{\prime}(\xi) \mathrm{d} \xi \\
& +\int_{0}^{1} \rho \mathrm{v}^{2} l^{-2}(1-\xi)^{2} \varphi_{i}(\xi) \varphi_{j}^{\prime \prime}(\xi) \mathrm{d} \xi \\
& -\int_{0}^{1} E A l^{-2} \varphi_{i}(\xi) \varphi_{j}^{\prime \prime}(\xi) \mathrm{d} \xi \\
& +\int_{0}^{1} m_{e}(g-\dot{v}) l^{-2} \varphi_{i}^{\prime}(\xi) \varphi_{j}^{\prime}(\xi) \mathrm{d} \xi \\
& +\frac{3 m v^{2}}{4} l^{-3} \varphi_{i}(1) \varphi_{j}(1)-\frac{m_{e} a}{2} l^{-2} \varphi_{i}(1) \varphi_{j}(1) \\
& +\int_{0}^{l} \rho l^{-1}(g-a) p_{j} \varphi_{i}(\xi) \varphi_{j}^{\prime}(\xi) \mathrm{d} \xi \\
& -\int_{0}^{1} \rho l^{-3}(1-\xi)(g-a) \varphi_{i}(\xi) \varphi_{j}^{\prime \prime}(\xi) \mathrm{d} \xi \\
& +E A l^{-2} \varphi_{j}^{\prime}(1) \varphi_{i}(1)-\int_{0}^{1} 0.5 E A l^{-4} e^{2} \varphi_{i}(\xi) \varphi_{j}^{\prime \prime}(\xi) \mathrm{d} \xi
\end{aligned}
$$

$$
\begin{aligned}
& k_{2 i j}=\left(\frac{3 \rho}{4} v^{2} l^{-2}-\frac{\rho a}{2} l^{-1}\right) \delta_{i j} \\
& -3 \int_{0}^{1} \rho v^{2} l^{-2}(1-\xi) \varphi_{j}^{\prime}(\xi) \varphi_{i}(\xi) \mathrm{d} \xi \\
& +\int_{0}^{1} \rho a l^{-1}(1-\xi) \varphi_{i}(\xi) \varphi_{j}^{\prime}(\xi) \mathrm{d} \xi \\
& +\int_{0}^{1} \rho v^{2} l^{-2}(1-\xi)^{2} \varphi_{j}^{\prime \prime}(\xi) \varphi_{i}(\xi) \mathrm{d}(\xi) \\
& +\int_{0}^{1} \rho l^{-1}(g-a) \varphi_{j}^{\prime}(\xi) \varphi_{i}(\xi) \mathrm{d} \xi \\
& +\int_{0}^{1} m_{e}(g-a) l^{-2} \varphi_{i}^{\prime}(\xi) \varphi_{j}^{\prime}(\xi) \mathrm{d} \xi \\
& -\int_{0}^{1} \rho l^{-3}(1-\xi)(g-a) \varphi_{j}^{\prime \prime}(\xi) \varphi_{i}(\xi) \mathrm{d} \xi \\
& -\int_{0}^{1} 1.5 E A e^{2} l^{-4} \varphi_{j}^{\prime \prime}(\xi) \varphi_{i}(\xi) \mathrm{d} \xi \\
& +\frac{3 m_{e} v^{2}}{4} l^{-3} \varphi_{i}(1) \varphi_{j}(1)-\frac{m_{e} a}{2} l^{-2} \varphi_{i}(1) \varphi_{j}(1) \\
& +k l^{-1} \varphi_{i}(1) \varphi_{j}(1)-\frac{1}{2} c v l^{-2} \varphi_{i}(1) \varphi_{j}(1) \\
& C P_{1 i}=\int_{0}^{l} 3 E A l^{-3.5} \varphi_{i}(\xi)\left[\sum_{j=1}^{n} \varphi_{j}^{\prime}(\xi) p_{j}\right]\left[\sum_{j=1}^{n} \varphi_{j}^{\prime \prime}(\xi) p_{j}\right] \mathrm{d} \xi \\
& +\int_{0}^{1} 1.5 E A l^{-5} \varphi_{i}(\xi)\left[\sum_{j=1}^{n} \varphi_{j}^{\prime}(\xi) p_{j}\right]^{2}\left[\sum_{j=1}^{n} \varphi_{j}^{\prime \prime}(\xi) p_{j}\right] \mathrm{d} \xi \\
& +\int_{0}^{l} 0.5 E A l^{-5} \varphi_{i}(\xi)\left[\sum_{j=1}^{n} \varphi_{j}^{\prime}(\xi) q_{j}\right]^{2}\left[\sum_{j=1}^{n} \varphi_{j}^{\prime \prime}(\xi) p_{j}\right] \mathrm{d} \xi \\
& +\int_{0}^{l} E A l^{-5} \varphi_{i}(\xi)\left[\sum_{j=1}^{n} \varphi_{j}^{\prime}(\xi) p_{j}\right]\left[\sum_{j=1}^{n} \varphi_{j}^{\prime}(\xi) q_{j}\right] \\
& {\left[\sum_{j=1}^{n} \varphi_{j}^{\prime \prime}(\xi) q_{j}\right] \mathrm{d} \xi+\int_{0}^{l} E A l^{-3.5} \varphi_{i}(\xi)\left[\sum_{j=1}^{n} \varphi_{j}^{\prime}(\xi) q_{j}\right]} \\
& {\left[\sum_{j=1}^{n} \varphi_{j}^{\prime \prime}(\xi) q_{j}\right] \mathrm{d} \xi-1.5 E A l^{-3.5}\left[\sum_{j=1}^{n} \varphi_{j}^{\prime}(1) p_{j}\right]^{2} \varphi_{i}(1)} \\
& -0.5 E A l^{-5}\left[\sum_{j=1}^{n} \varphi_{j}^{\prime}(1) p_{j}\right]^{3} \varphi_{i}(1) \\
& -0.5 E A l^{-3.5}\left[\sum_{j=1}^{n} \varphi_{j}^{\prime}(1) q_{j}\right]^{2} \varphi_{i}(1) \\
& -0.5 E A l^{-5} \sum_{j=1}^{n} \varphi_{j}^{\prime}(1) p_{j}\left[\sum_{j=1}^{n} \varphi_{j}^{\prime}(1) q_{j}\right]^{2} \varphi_{i}(1)
\end{aligned}
$$




$$
\begin{aligned}
& C P_{2 i}=\int_{0}^{l} E A l^{-3.5} \varphi_{i}(\xi)\left[\sum_{j=1}^{n} \varphi_{j}^{\prime}(\xi) q_{j}\right]\left[\sum_{j=1}^{n} \varphi_{j}^{\prime \prime}(\xi) p_{j}\right] \mathrm{d} \xi \\
& +\int_{0}^{l} 1.5 E A l^{-5} \varphi_{i}(\xi)\left[\sum_{j=1}^{n} \varphi_{j}^{\prime}(\xi) q_{j}\right]^{2}\left[\sum_{j=1}^{n} \varphi_{j}^{\prime \prime}(\xi) q_{j}\right] \mathrm{d} \xi \\
& +\int_{0}^{l} 0.5 E A l^{-5} \varphi_{i}(\xi)\left[\sum_{j=1}^{n} \varphi_{j}^{\prime}(\xi) p_{j}\right]^{2}\left[\sum_{j=1}^{n} \varphi_{j}^{\prime \prime}(\xi) q_{j}\right] \mathrm{d} \xi \\
& +\int_{0}^{l} E A l^{-5} \varphi_{i}(\xi)\left[\sum_{j=1}^{n} \varphi_{j}^{\prime}(\xi) p_{j}\right]\left[\sum_{j=1}^{n} \varphi_{j}^{\prime}(\xi) q_{j}\right] \\
& {\left[\sum_{j=1}^{n} \varphi_{j}^{\prime \prime}(\xi) p_{j}\right] \mathrm{d} \xi+\int_{0}^{l} E A l^{-3.5} \varphi_{i}(\xi)\left[\sum_{j=1}^{n} \varphi_{j}^{\prime}(\xi) p_{j}\right]} \\
& {\left[\sum_{j=1}^{n} \varphi_{j}^{\prime \prime}(\xi) q_{j}\right] \mathrm{d} \xi-\sum_{j=1}^{n} E A l^{-3.5}\left[\sum_{i=1}^{n} \varphi_{i}^{\prime}(1) p_{i}\right]} \\
& {\left[\sum_{i=1}^{n} \varphi_{i}^{\prime}(1) q_{i}\right] \varphi_{i}(1)-0.5 E A l^{-5}\left[\sum_{i=1}^{n} \varphi_{i}^{\prime}(1) p_{i}\right]^{2}} \\
& {\left[\sum_{i=1}^{n} \varphi_{i}^{\prime}(1) q_{i}\right] \varphi_{i}(1)-0.5 E A l^{-5}\left[\sum_{i=1}^{n} \varphi_{j}^{\prime}(1) q_{i}\right]^{3} \varphi_{i}(1)} \\
& -\int_{0}^{l} 3 E A e l^{-4.5} \varphi_{i}(\xi)\left[\sum_{j=1}^{n} \varphi_{j}^{\prime}(\xi) q_{j}\right]\left[\sum_{j=1}^{n} \varphi_{j}^{\prime \prime}(\xi) q_{j}\right] \mathrm{d} \xi \\
& f_{1 i}=m g l^{-0.5} \varphi_{i}(1)-\int_{0}^{1} E A l^{-3} e \varphi_{i}(\xi)\left[\sum_{j=1}^{n} \varphi_{j}^{\prime \prime}(\xi) q_{j}\right] \mathrm{d} \xi \\
& -\int_{0}^{l} \operatorname{EAel}^{-4.5} \varphi_{i}(\xi)\left[\sum_{j=1}^{n} \varphi_{j}^{\prime \prime}(\xi) q_{j}\right]\left[\sum_{j=1}^{n} \varphi_{j}^{\prime}(\xi) p_{j}\right] \mathrm{d} \xi \\
& -\int_{0}^{1} E A l^{-4.5} \varphi_{i}(\xi) e\left[\sum_{j=1}^{n} \varphi_{j}^{\prime \prime}(\xi) p_{j}\right]\left[\sum_{j=1}^{n} \varphi_{j}^{\prime}(\xi) q_{j}\right] \mathrm{d} \xi \\
& f_{2 i}=\int_{0}^{1} \varphi_{i}(\xi) l^{-0.5}\left[\rho(g-a) e-\rho e^{\prime \prime}(1-\xi) l\right. \\
& \left.+2 \rho v e^{\prime}(1-\xi)-2 \rho e v^{2} l^{-1}(1-\xi)+\rho a e(1-\xi)\right] \mathrm{d} \xi \\
& -\int_{0}^{1} E A e l^{-3}\left[\sum_{j=1}^{n} \varphi_{j}^{\prime \prime} p_{j}\right] \mathrm{d} \xi \\
& -\int_{0}^{1} E A e l^{-4.5}\left[\sum_{j=1}^{n} \varphi_{j}^{\prime \prime} p_{j}\right] \\
& {\left[\sum_{j=1}^{n} \varphi_{j}^{\prime} p_{j}\right] \varphi_{i}(\xi) \mathrm{d} \xi+l^{-0.5} k s \varphi_{i}(1)} \\
& +l^{-0.5} c \dot{s} \varphi_{i}(1)
\end{aligned}
$$

where $\delta_{i j}$ was the Kronecker delta defined by $\delta_{i j}=0$ if $i \neq j$ and $\delta_{i j}=1$ if $i=j$.

\section{References}

Cao GH (2009) Impact dynamic behaviors of mine hoisting rope during loading process. China University of Mining \& Technology, Xuzhou (in Chinese with English Abstract)

Kaczmarczyk S, Ostachowicz W (2003a) Transient vibration phenomena in deep mine hoisting cables. Part 1: mathematical mode. J Sound Vib 262:219-244

Kaczmarczyk S, Ostachowicz W (2003b) Transient vibration phenomena in deep mine hoisting cables. Part 2: numerical simulation of the dynamic response. J Sound Vib 262:245-289

Li ZF (2010) Study on the vibration characteristic and typical fault diagnosis of mine hoist system. China University of Mining \& Technology, Xuzhou (in Chinese with English Abstract)

Qin D (2007) Study on safety of coal mine vertical friction hoisting system based on dynamic. Hefei University of Technology, Hefei (in Chinese with English Abstract)

Sandilo SH, van Horssen WT (2014) On variable length induced vibrations of a vertical string. J Sound Vib 333:2432-2449

Zhang P (2007) Theoretic and test research on dynamic behaviors of high speed elevator suspended system. Doctor's thesis, Shanghai Jiao Tong University (in Chinese with English Abstract)

Zhu WD, Chen Y (2005) Forced response of translating media with variable length and tension: application to high-speed elevators. Proc Inst Mech Eng, Part K 219(1):35-53

Zhu WD, Ni J (2000) Energetics and stability of translating media with an arbitrary varying length. J Vib Acoust, Trans ASME 122(3):295-304

Zhu WD, Ni J, Huang J (2001) Active control of translating media with arbitrarily varying length. J Vib Acoust, Trans ASME 123(3):347-358 